\title{
Prevalencia de queilitis actínica en trabajadores expuestos a radiación ultravioleta en Talca, Chile
}

\section{Prevalence of actinic cheilitis in Chilean workers exposed to ultraviolet radiation}

\author{
Orozco $\mathrm{P}^{1}$, Vásquez $\mathrm{S}^{2,5}$, Venegas $\mathrm{B}^{3,5}$, Rivera $\mathrm{C}^{4,5}$
}

\begin{abstract}
RESUMEN
El objetivo de esta investigación es determinar la prevalencia de queilitis actínica (QA) y factores asociados en trabajadores expuestos a radiación UV solar en la ciudad de Talca, Chile. Se examinaron 151 trabajadores, donde un $16.6 \%$ presentó QA. Todas las lesiones se presentaron en el labio inferior. El $40 \%$ de los casos poseía una edad entre 50 y 59 años y el $96 \%$ de los pacientes diagnosticados trabajaba más de 6 horas diarias expuestos al sol. El $56 \%$ de los diagnósticos de QA presentaron un historial 20 o más años totales de exposición a radiación UV por motivos laborales. El $56 \%$ de los casos de QA presentaron antecedentes de hábito de fumar. Los principales signos presentes en los casos de QA fueron la sequedad (100\%), esfumación del margen del bermellón (88\%) y el edema labial (84\%). La mayoría de las lesiones fueron asintomáticas (60\%). Rev. Clin. Periodoncia Implantol. Rehabil. Oral Vol. 6(3); 127-129, 2013.
\end{abstract}

Palabras clave: Queilitis actínica, estudio de prevalencia, radiación ultravioleta.

\section{ABSTRACT}

The objective of this research is to determine the prevalence of actinic cheilitis (AC) and associated factors in workers exposed to solar UV radiation in the city of Talca (Chile). A total of 151 workers, of which $16.6 \%$ exhibited AC, were examined. All lesions appeared on the lower lip. The $40 \%$ of cases aged between $50-59$ years and $96 \%$ of patients diagnosed worked up to 6 hours daily exposed to the sun. $56 \%$ of the diagnoses of AC had a history of 20 or more years of exposure to UV radiation for work. $56 \%$ of cases of AC had a history of smoking. Major signs present in the AC cases were the dryness (100\%), blurred demarcation between the lip vermilion border and the skin ( $88 \%)$ and labial edema (84\%). Most lesions were asymptomatic $(60 \%)$.

Rev. Clin. Periodoncia Implantol. Rehabil. Oral Vol. 6(3); 127-129, 2013.

Key words: Actinic cheilitis, prevalence studies, ultraviolet radiation.

\section{INTRODUCCIÓN}

Entre las alteraciones de la mucosa oral se encuentras los trastornos orales potencialmente malignizantes (TOPMs), que otorgan un mayor riesgo para el desarrollo de cáncer oral(1-3). La queratosis actínica de la piel corresponde a una patología inducida por radiación ultravioleta (UV), cuya lesión análoga en el labio constituye un TOPM denominado queilitis actínica $(\mathrm{QA})^{(4)}$, la que principalmente se manifiesta labio inferior de personas género masculino con constante y prolongada exposición a radiación UV solar ${ }^{(5)}$. La QA posee una baja tasa de malignización a carcinoma de células escamosas de la cavidad oral (entre el $1 \%$ y el $3 \%$ ), sin embargo, cuando se transforma en cáncer tiene alto poder de metástasis ${ }^{(6-9)}$. Dentro de los factores asociados a QA se encuentran individuos con fototipo ligeramente pigmentados, según la clasificación de Fitzpatrick entre tipo I y IV ${ }^{(10)}$, además se considera el consumo de tabaco como un importante factor de riesgo para su desarrollo(11,12).

El diagnóstico de la QA es sencillo y se obtiene por la correlación de la anamnesis con los hallazgos clínicos en un paciente bajo situación de riesgo ${ }^{(1)}$. Además, la presencia de queratosis solar en otras áreas expuestas (cara, nariz, cuero cabelludo, orejas) refuerza el diagnóstico. Debido a su condición precancerosa, las lesiones que persisten o que no responden a medidas terapéuticas deben ser biopsiadas ${ }^{(13)}$.

En Chile existe información previa respecto a prevalencia de QA, sin embargo, la información es escasa, y en algunos casos los datos fueron obtenidos a partir de grupos que no reúnen los factores de riesgo para el desarrollo de este TOPM, por lo tanto, el objetivo de esta investigación es determinar la prevalencia de QA y sus factores asociados en personas expuestas a UV durante su jornada laboral en una ciudad de Chile.

\section{MATERIALES Y MÉTODOS}

Se realizó un estudio descriptivo de prevalencia en la ciudad de Talca, Región del Maule, Chile, entre Agosto y Septiembre de 2011. Se seleccionó una muestra por conveniencia de 151 sujetos (84 hombres y 67 mujeres) expuestos a radiación UV durante su jornada laboral (parquímetros y personal de mantención de áreas verdes). Mediante firma de consentimiento informado los individuos manifestaron participar de forma voluntaria e informada. Todos los procedimientos fueron aprobados por el Comité de Bioética de la Universidad de Talca y siguen los lineamientos de Bioética e Investigación Científica en Seres Humanos y Animales (CONICYT, Chile, 2006).

Para determinar la presencia de QA se realizó un examen clínico en instalaciones de trabajo de los sujetos bajo estudio realizado un profesional previamente calibrado (Kappa intra-examinador 0.84) que registró la presencia de factores de riesgo (tabaco, exposición solar y

\footnotetext{
1. BDS. Centro de Salud Familiar CESFAM. Departamento Comunal de Salud, Ilustre Municipalidad de Pencahue. Pencahue, Chile.

2. MSc. Unidad de Patología Oral. Departamento de Estomatología. Facultad de Ciencias de la Salud, Universidad de Talca. Chile.

3. phD. Unidad de Patología Oral. Departamento de Estomatología. Facultad de Ciencias de la Salud, Universidad de Talca. Chile.

4. MSc. Unidad de Histología y Embriología. Departamento de Ciencias Básicas Biomédicas, Universidad de Talca. Chile.

5. Clínica de Patología Oral de la Región del Maule. CreoSalud. Talca, Chile.
} 
fototipo), utilización de elementos protectores (sombrero, protector solar o labial), presencia/ausencia de QA y características clínicas de la lesión presente en los labios de los trabajadores. Junto con la inspección visual se utilizaron espejos dentales, guantes de examen, mascarillas y luz artificial.

El diagnóstico clínico se acompañó con el registro de presencia/ ausencia de manifestaciones de la patología como sequedad, aumento de volumen, esfumación de margen del bermellón, áreas atróficas, lesiones escamosas, eritema, ulceración, pliegues marcados, costras, áreas con manchas blancas o palidez, además de síntomas como ardor o picazón. Los casos diagnosticados fueron derivados a la Clínica de Diagnóstico de Patología Oral de la Universidad de Talca, Región del Maule, Chile, para indicación de tratamiento.

\section{RESULTADOS}

La prevalencia de trabajadores diagnosticados con QA en la muestra es de $16.6 \%(n=25)$. Todas las lesiones se presentaron en el labio inferior, mayoritariamente en el centro $60 \%(n=15)$, seguido por la mucosa labial $28 \%(n=7)$ y el margen del bermellón $12 \%(n=3)$.

En cuanto a distribución por sexo de pacientes diagnosticados con QA, se observó que el $64 \%$ fueron hombres $(n=16)$ y el $36 \%$ mujeres $(n=9)$. El $40 \%$ de los casos posee una edad entre 50 y 59 años $(n=10)$ y un $20 \%$ se encuentra entre los 20 y 29 años $(n=5)$ (Tabla 1 ).

La Tabla 1 muestra que el $96 \%$ de los pacientes diagnosticados trabaja más de 6 horas diarias expuestos al sol $(n=24)$. Cabe destacar que el $56 \%$ de los pacientes $(n=16)$ con QA presenta 20 o más años totales de exposición a radiación UV por motivos laborales.

El fototipo predominante en trabajadores diagnosticados corresponde a pigmentación intermedia en $84 \%$ de los casos $(n=21)$ y $16 \%$ ligeramente pigmentados $(n=4)$.

Respecto al hábito de fumar el $44 \%(n=11)$ de los pacientes diagnosticados con QA se manifiesta como no fumador, el $36 \%$ actualmente fuma $(n=9)$ y el $20 \%$ son ex- fumadores (Tabla 1$)$.

Los elementos protectores como sombrero y bloqueador solar son utilizados en el $80 \%$ de los trabajadores diagnosticados con QA $(n=20)$, mientras que un $20 \%$ no los utiliza $(n=5)$.

La Figura 1 muestra pacientes con diagnóstico de QA. En la prevalencia de signos y síntomas se encontró que sequedad labial se encuentra en la totalidad de los casos diagnosticados $(n=25)$. Este se acompañó principalmente de esfumación del margen del bermellón en el $88 \%$ $(n=22)$ y aumento de volumen labial en el $84 \%$ de los casos $(n=21)$, pliegues marcados en un $76 \%$ ( $n=19)$ (Tabla 2). Las lesiones de QA se presentaron mayormente asintomáticas en $60 \%$ de los diagnosticados $(n=15)$.
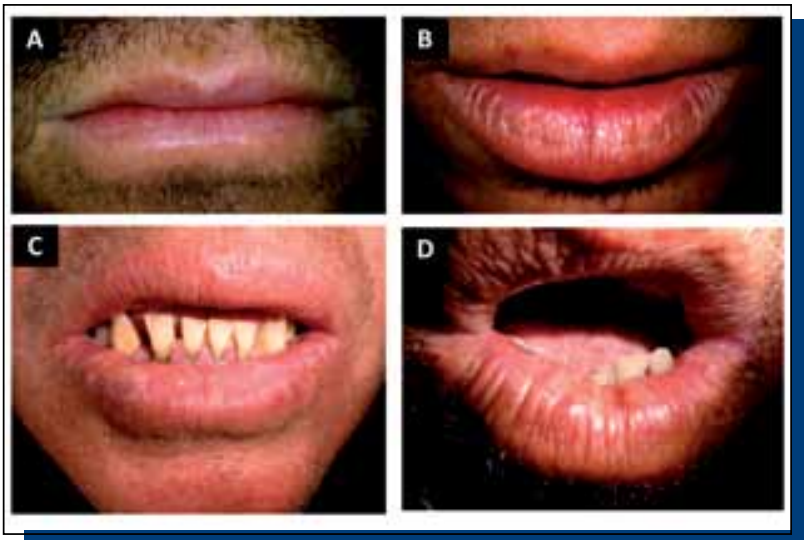

Figura 1. Queilitis actínica en trabajadores expuestos a radiación UV solar en Talca, Chile. Sequedad, edema y comienzo de esfumación del labio inferior (A). Sequedad, pliegues labiales marcados y lesiones escamosas (B). Sequedad, edema, eritema, placas blancas y pequeñas zonas de ulceración (C). Sequedad, edema, eritema, pliegues labiales marcados y costra (D).
Tabla 1. Distribución y frecuencia de queilitis actínica según sexo, años de exposición UV y hábito de fumar.

\begin{tabular}{|l|c|c|c|c|}
\hline \multirow{2}{*}{} & \multicolumn{4}{|c|}{ Queilitis Actínica } \\
\cline { 2 - 5 } & \multicolumn{3}{|c|}{ Presente } & \multicolumn{2}{c|}{ Ausente } \\
\cline { 2 - 5 } & $\mathrm{n}$ & $\%$ & $\mathrm{n}$ & $\%$ \\
\hline Sexo & \multicolumn{5}{|c|}{} \\
\hline Hombre & 16 & 64 & 68 & 54 \\
\hline Mujer & 9 & 36 & 58 & 46 \\
\hline Años de Exposición UV & & 20 & 48 & 38.1 \\
\hline $0-9$ & 5 & 24 & 50 & 39.7 \\
\hline $10-19$ & 6 & 56 & 28 & 22.2 \\
\hline 20 o más & 14 & 44 & 59 & 46.8 \\
\hline Hábito de Fumar & & 20 & 1 & 0.8 \\
\hline No fuma & 11 & 36 & 66 & 52.4 \\
\hline Ex fumador & 9 & & & \\
\hline Fumador & & & & \\
\hline
\end{tabular}

Tabla 2. Distribución y frecuencia de los signos y síntomas presentes en los casos de queilitis actínica.

\begin{tabular}{|l|c|c|}
\hline \multicolumn{1}{|c|}{ Signos y Síntomas } & $\mathbf{n}$ & $\%$ \\
\hline Sequedad & 25 & 100 \\
\hline Esfumación del borde del bermellón & 22 & 88 \\
\hline Edema & 21 & 84 \\
\hline Pliegues labiales marcados & 19 & 76 \\
\hline Lesiones escamosas & 13 & 52 \\
\hline Atrofia & 12 & 48 \\
\hline Manchas o placas blancas & 11 & 44 \\
\hline Ardor o prurito & 10 & 40 \\
\hline Costras & 4 & 16 \\
\hline Eritema & 4 & 16 \\
\hline Ulceración & 2 & 8 \\
\hline
\end{tabular}

\section{DISCUSIÓN}

La prevalencia encontrada en esta investigación (16.6\%) difiere a otros estudios, por ejemplo previamente en Talca (Chile) se obtuvo una prevalencia de $1.6 \%^{(7)}$ y en un estudio previo que buscó la prevalencia de lesiones de la mucosa oral en 885 sujetos sobre 65 años en Santiago (Chile), la QA representó el 0.9\% (8 casos) $)^{(14)}$. Previamente, en una muestra de 566 sujetos (pescadores artesanales) de Valparaíso (Chile), la presencia de QA fue de un $43 \%$, lo que es considerablemente superior a nuestros hallazgos, en un escenario que corresponde a trabajadores expuestos al sol(15). En trabajadores agrícolas en Brasil la prevalencia fue superior, encontrándose QA en un $39.6 \%{ }^{(8)}$

La variación en prevalencia de QA encontrada en la literatura puede deberse por las disímiles condiciones bajo las cuales fueron realizados cada uno de estos estudios así como también a las condiciones particulares de cada muestra y ambiente de exposición.

La lesión se manifiesta en el $100 \%$ de los casos en el labio inferior lo que coincide con la bibliografía donde se indica que la lesión se ubica preferentemente en el labio inferior ${ }^{(1)}$. Previemente en Chile, datos de un estudio retrospectivo que describió las características clínicas y patológicas del cáncer labial y TOPMs de 232 casos del Instituto de Referencia de Patología Oral de la Universidad de Chile (IREPO), muestran que de 100 casos de QA, el labio inferior estuvo afectado en 96 de ellos ${ }^{(16)}$.

Según género, los casos diagnosticados se presentan mayoritariamente en hombres, lo que coincide con hallazgos previos ${ }^{(17)}$. Datos del IREPO muestran que el $64 \%$ de los casos de QA se presentó 
en hombres, lo que es idéntico a nuestras cifras, estableciendo una relación hombre-mujer de 1.77:1(16).

La relativa protección de la mujer frente a la QA podría atribuirse a dos causas: el uso de lápiz labial (que puede constituir un factor de protección solar) ${ }^{(16)}$ y la realización de menores actividades laborales al aire libre, lo que las hace menos susceptibles a acumular los efectos del daño actínico.

El $40 \%$ de los casos posee una edad entre 50 y 59 años $(n=10)$ y un $20 \%$ se encuentra entre los 20 y 29 años $(n=5)$. Datos previos desde una muestra chilena, indican que los pacientes sobre 45 años presentan una mayor incidencia de $Q \mathrm{~A}^{(15)}$.

La edad de manifestación del TOPM fue mayoritariamente en la quinta década de edad, lo que se condice con publicaciones previas ${ }^{(16)}$. Resultados publicados previamente muestran que los individuos que se exponen habitualmente al sol desde edades tempranas de la vida, sumado además, con historial de exposición solar acumulada durante la vida laboral, los hace más susceptibles de desarrollar $\mathrm{QA}^{(12)}$. Nuestros resultados además coinciden con un estudio donde la población afectada está integrada principalmente por trabajadores entre la quinta y sexta década de vida $^{(9)}$.

En Chile, Nocolini et al. (1989), concluyen que existe una relación directa entre los años de exposición solar y la severidad de las alteraciones labiales que pueden ser encontradas en pacientes en riesgo, este antecedente se suma a nuestros resultados, que muestran que la mayoría de los casos de QA diagnosticados presentaron una exposición de 20 o más años a radiación actínica, lo que fortalece el argumento que el daño por radiación UV es acumulativo, manifestando en forma progresiva signos clínicos en una lesión potencialmente malignizante.

Los trabajadores diagnosticados trabajan más de 6 horas diarias expuestos a radiación UV y durante ambas jornadas (mañana y tarde) en el $100 \%$ de los casos. Lo anterior concuerda con un estudio, el cual afirma que trabajadores con más horas diarias de exposición a radiación solar tienen mayor posibilidad de desarrollar QA. La radiación UV es más intensa entre las 11 y las 16 horas, periodo en el que trabajadores desarrollan sus labores, lo que favorece la aparición de $\mathrm{QA}^{(11)}$.

Respecto al fototipo de los trabajadores con QA se obtuvo que la mayoría de ellos posee pigmentación intermedia. Estos datos se contradicen con resultados previos donde principalmente se asocia la presencia de QA en fototipos ligeramente pigmentados ${ }^{(8)}$. Es probable que la presencia de QA se asocie además a una características fenotípicas como pelo rubio o pelirrojo, ojos azules o verdes y piel clara $^{(15)}$

El 56\% de los pacientes con QA tiene historial de hábito de fumar, considerando tanto fumadores como exfumadores. Diversos estudios manifiestan asociación entre fumar y mayor probabilidad de manifestación de $\mathrm{QA}^{(10,18)}$. Sin embargo, otro estudio no lo asocia como factor de riesgo asociado al desarrollo de la lesión(8).

La mayoría de los trabajadores diagnosticados con QA utilizaban elementos protectores, pese a la protección el TOPM estaba presente. Sería necesario profundizar en la antigüedad de la protección, ya que en años recientes la población considera la exposición solar como riesgo para el desarrollo de diversos cánceres. Ningún estudio establece relación significativa entre la presencia de estas variables con el desarrollo de $\mathrm{QA}^{(19,20)}$. Existe evidencia que informa el efecto preventivo de la aplicación diaria de fotoprotectores para prevenir la aparición de la lesión ${ }^{(21,22)}$.

Las manifestaciones clínicas encontradas muestran que la sequedad de labio se presento en todos los casos de QA diagnosticadas, aspecto que coincide en comparación con una publicación previa ${ }^{(9)}$.

La mayoría de las lesiones fueron asintomáticas. Los trabajadores con QA que relataron sintomatología (picazón o ardor) en el $40 \%$ de casos, similar al encontrado en un estudio donde el $34.5 \%$ presenta sintomatología ${ }^{(17)}$. Previamente se ha informado que la expresión crónica QA cursa con alteraciones de mayor severidad en el bermellón ${ }^{(15)}$.

Este estudio pretende contribuir sobre los aspectos epidemiológicos de la $Q A$, especialmente los efectos nocivos de la radiación UV, sobre todo para las personas sometidas a exposición intensa y crónica de tipo laboral. Es importante intensificar las medidas educativas y preventivas, así como asegurar las acciones curativas adecuadas para esta población.

Aunque la QA debería ser de fácil diagnóstico en la clínica odontológica, muchas veces es pasada por alto al examen rutinario y por tanto creemos que es relevante el estudio y conocimiento de este TOPM, con el fin de el cirujano dentista pueda intervenir precozmente en un proceso de malignización que lleve posiblemente a un cáncer en nuestro territorio de praxis.

\section{AGRADECIMIENTOS Y CONFLICTO DE INTERESES}

Los autores agradecen a la Dirección de Investigación (DI) de la Universidad de Talca y a la Unidad de Investigación del Centro Odontológico CreoSalud, Talca. El manuscrito no presenta conflicto de intereses.

\section{REFERENCIAS BIBLIOGRÁFICAS}

1. Huber MA. White oral lesions, actinic cheilitis, and leukoplakia: Confusions in terminology and definition. Facts and controversies. Clin Dermatol, 2010; 28(3): 262-268. 2. Lucas RM, McMichael AJ, Armstrong BK, Smith WT. Estimating the global disease burden due to ultraviolet radiation exposure. Int J Epidemiol, 2008; 37(3): 654-667.

3. Martínez A, Spencer M, Borlando J, Flores M, Rojas I. E-cadherin and c-Met expression in actinic cheilits and lip squamous cell carcinoma. Rev Clin Periodoncia Implantol Rehabil Oral, 2011; 4(3): 122-125.

4. van der Waal I. Potentially malignant disorders of the oral and oropharyngeal mucosa; terminology, classification and present concepts of management. Oral Oncol, 2009; 45(4): 317-323.

5. Wood NH, Khammissa R, Meyerov R, Lemmer J, Feller L. Actinic cheilitis: A case report and a review of the literature. Eur J Dent, 2011; 5(1): 101.

6. Bentley JM, Barankin B, Lauzon GJ. Paying more than lip service to lip lesions. Can Fam Physician, 2003; 49(9): 1111-1116.

7. Acevedo KI. Prevalencia de lesiones potencialmente malignizantes de la mucosa oral en adultos mayores de los consultorios Dr. José Dionisio Astaburuaga y Dr. Julio Contardo, Talca, 2006 [tesis de grado]. Talca: Universidad de Talca, Escuela de Odontología; 2006.

8. Junqueira J, Bönecker M, Furuse C, Morais PC, Flório F, Cury P et al. Actinic cheilitis among agricultural workers in Campinas, Brazil. Community Dent Health, 2011; 28(1): 60 .

9. Cavalcante ASR, Anbinder AL, Carvalho YR. Actinic cheilitis: Clinical and histological features. J Oral Maxil Surg, 2008; 66(3): 498-503.

10. López EPM, Minarro-Del Moral R, Martinez-Garcia C, Zanetti R, Rosso S, Serrano $S$ et al. Lifestyles, environmental and phenotypic factors associated with lip cancer: A case-control study in southern Spain. Br J Cancer, 2003; 88(11): 1702-1707.

11. Puga PM. Parámetros de riesgo de la queilitis actínica crónica. Tesis doctoral. Granada: Universidad de Granada, Departamento de Estomatología; 2011.
12. Salasche SJ. Epidemiology of actinic keratoses and squamous cell carcinoma $J A A D, 2000 ; 42(1 \mathrm{Pt} 2): 4$

13. Michaell ASHTGDT. The patient with actinic cheilosis. Gen Dent, 2006; 6: 274-282. 14. Espinoza I, Rojas R, Aranda W, Gamonal J. Prevalence of oral mucosal lesions in elderly people in Santiago, Chile. J Oral Pathol Med, 2003; 32(10): 571-575.

15. Nicolini S, Ascorra C, Guzman C, Latife AV. Queilitis actinica en pescadores artesanales de la Quinta Región: Prevalencia y aspectos histopathologicos asociados. Odont Chil, 1989; 37(1): 169-174. Epub 1989/04/01.

16. Ochsenius RG, Ormeño QA, Godoy RL, Rojas R. Estudio retrospectivo de 232 casos de cáncer y precáncer de labio en pacientes chilenos: Correlación clínicohistológica. Rev Med Chile, 2003; 131(1): 60-66.

17. Wood NH, Khammissa R, Meyerov R, Lemmer J, Feller L. Actinic cheilitis: A case report and a review of the literature. Eur $J$ Dent, 2011; 5(1): 101-106. Epub 2011/01/14

18. Campisi G, Margiotta V. Oral mucosal lesions and risk habits among men in an Italian study population. J Oral Pathol Med, 2001; 30(1): 22-28.

19. Morison WL. Photoprotection by clothing. Dermatol Ther, 2003; 16(1): 16-22.

20. Rigel D. Photoprotection: A $21^{\text {st }}$ century perspective. Br J Dermatol, 2002 146(s61): 34-37.

21. Darlington S, Williams G, Neale R, Frost C, Green A. A randomized controlled trial to assess sunscreen application and beta carotene supplementation in the prevention of solar keratoses. Arch Dermatol, 2003; 139(4): 451

22. Drolet BA, Connor MJ. Sunscreens and the prevention of ultraviolet radiationinduced skin cancer. J Dermatol Surg Oncol, 1992; 18(7): 571-576. Epub 1992/07/01 